\title{
Explicit formulas for Killing magnetic curves in Heisenberg group*
}

\author{
Khadidja Derkaoui ${ }^{1 \dagger}$ and Fouzi Hathout ${ }^{2}$ \\ ${ }^{1}$ Department of mathematics, university of Chlef, Algeria \\ Email: derkaouikhdidja248@hotmail.com \\ ${ }^{2}$ Department of mathematics, university of Saïda, Algeria \\ Email: f.hathout@gmail.com
}

\begin{abstract}
In this paper, We present the geometry three dimensional Heisenberg group $\left(\mathbb{H}_{3}, g\right)$ and its geodesics curves. After, we study the Killing magnetic curves and some geodesic Killing magnetic curves with its explicit formulas for such curves.
\end{abstract}

Key words: Heisenberg group; geodesic Killing magnetic curves; Killing vector fields; Killing magnetic curves.

MSC: 53A04, 53C25

\section{Introduction}

In physics, particularly in electromagnetism, the trajectory of charged particles moving under the action of a magnetic fields makes an important research topic. In geometry, this trajectory in any manifold is known as a magnetic curve.

On a Riemannian manifold, the magnetic curve $\gamma$ is modeled as a solution of two order differential equation $\nabla_{\gamma^{\prime}} \gamma^{\prime}=\varphi\left(\gamma^{\prime}\right)$ known as LORENTZ equation, where $\varphi$ is $(1,1)$-tensor field that present the LORENTz force associated to the magnetic fields $F$. The magnetic curve generalise the geodesic curves in following way: When the particles move under the absence of the magnetic fields so freely only under the influence of gravity (i.e. $\varphi \equiv 0$ ), the LORENTZ equation is exactly the geodesic equation $\nabla_{\gamma^{\prime}} \gamma^{\prime}=0$.

${ }^{*}$ The paprer was supported by Laboratory of fundamental and applied mathematics, University of Oran

${ }^{\dagger}$ Corresponding author 
The magnetic curve was studied intensively in different kind of manifolds in Riemannian, Lorentzian and generally in pseudo-Riemannian concept. (See [1], 6], 7], [18])

Furthermore, if the magnetic fields $F$ corresponds to a killing vector, then the trajectory of particles moving under the action of $F$ is called Killing magnetic curve.

Interesting results on Killing magnetic curves are given in Euclidian 3space $([9])$, Minkowski 3-space $([8]), \mathbb{S}^{2} \times \mathbb{R}([17]), S L(2, \mathbb{R})([10])$, Sol Space ([12]), warped product manifold $([15])$, almost paracontact manifold ([5]), Almost Cosymplectic Sol Space ([11]) and in Walker manifolds ([2]).

The framework of the paper is to study the Killing magnetic curves in 3-dimensional Heisenberg group.

The contents of this study is the following: we present in the second section a general notions and definitions of killing magnetic curves.

The section 3 is devoted to the geometry of Heisenberg group and its contact structure.

Finally in the section 4, we classifies fourth kind of Killing magnetic curves, we give its explicit parametric formulas at each kind in different subsections and we close it with figures presented in Euclidian space.

\section{Preliminaries}

On Riemannian manifold $(M, g)$, the magnetic curves is a trajectories of the charged particles moving under the action of the magnetic fields $F$ which can be represented by a closed 2 -form

$$
F(X, Y)=g(\varphi(X), Y)
$$

where $X, Y$ are vector fields on $(M, g)$ and $\varphi$ is skew-symmetric $(1,1)$-tensor field. that present the LoRENTz force associated to $F$. For a regular curve $\gamma: I \subset \mathbb{R} \rightarrow(M, g), \gamma$ is called magnetic curve if

$$
\nabla_{\mathbf{t}} \mathbf{t}=\varphi(\mathbf{t})
$$

known as the Lorentz equation, where $\nabla$ is the Levi-Civita connection associated to $g$ and $\mathbf{t}=\gamma^{\prime}$ is the speed vector of $\gamma$. The magnetic curve generalise the notion of geodesic curve under arc-length parametrization.

From the property of magnetic curve $\gamma$

$$
\frac{d}{d t} g\left(\gamma^{\prime}, \gamma^{\prime}\right)=0
$$


$\gamma$ has a constant speed vector. In particular, if $\gamma$ is parameterized by the arc length, then $\gamma$ is called normal magnetic curve.

We call $K$ a Killing vector field on $M$ if it satisfy the Killing equation

$$
g\left(\nabla_{X} K, Y\right)+g\left(\nabla_{Y} K, X\right)=0
$$

for every vector fields $X, Y$ on $M$.

We define on $\mathrm{M}$ the cross product of two vector fields $X, Y$ on $M$ as

$$
g(X \times Y, Z)=\operatorname{dvg}(X, Y, Z)
$$

for all vector fields $Z$ on $M$ and $d v g$ denotes the volume form on $M$.

Let $F_{K}=i_{K} d v g$ be the Killing magnetic field corresponding to the Killing vector field $K$ on $M$, where $i$ is inner product. Then, the $(1,1)$ tensor fields corresponding to the Lorentz force of $F_{K}$ is

$$
\gamma(X)=K \times X
$$

Then, we can rewrite the Lorentz equation Eq.(2) as

$$
\nabla_{\mathbf{t}} \mathbf{t}=K \times \mathbf{t}
$$

and the it solution is called Killing magnetic curves corresponding to the killing vector fields $K$.

In the sequel, to simplify, we call this curve a $K$-magnetic curve.

\section{$3 \quad$ Geometry structure of $\mathbb{H}_{3}$}

The Heisenberg group $\mathbb{H}_{3}$ is a quasi-abelian Lie group diffeomorphic to $\mathbb{R}^{3}$ and it has the standard representation in $G L(3, \mathbb{R})$ as

$$
\mathbb{H}_{3}=\left\{\left(\begin{array}{ccc}
0 & x & z \\
0 & 0 & y \\
0 & 0 & 0
\end{array}\right) \mid(x, y, z) \in \mathbb{R}^{3}\right\}
$$

endowed with the multiplication

$$
\left(x_{1}, y_{1}, z_{1}\right)\left(x_{2}, y_{2}, z_{2}\right)=\left(x_{1}+x_{2}, y_{1}+y_{2}, z_{1}+z_{2}-x_{1} y_{2}\right) .
$$

The invariant Riemannian metric with respect to the left-translations corresponding to that multiplication is denoted by

$$
g=\frac{1}{\lambda^{2}} d x^{2}+d y^{2}+(x d y+d z)^{2} .
$$


where $\lambda$ is a strictly positive real number. All left-invariant Riemannian metric on the $\mathbb{H}_{3}$ is isometric to the metric $g$.

The Levi-Civita connection $\nabla$ of the metric $g$ with respect to the leftinvariant orthonormal basis

$$
e_{1}=\partial y-x \partial z, \quad e_{2}=\lambda \partial x, \quad e_{3}=\partial z,
$$

with dual basis

$$
\omega^{1}=d y ; \quad \omega^{2}=\frac{1}{\lambda} d x ; \quad \omega^{3}=x d y+d z
$$

are given by

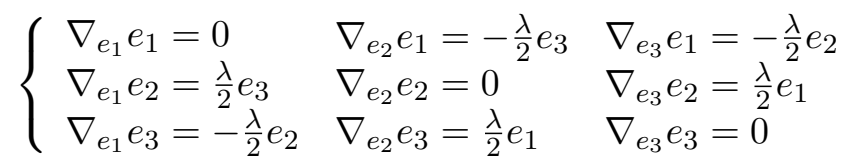

The Lie bracket of the base $\left(e_{i}\right)_{i=\overline{1,3}}$ are given by the following identities

$$
\left[e_{1}, e_{2}\right]=\lambda e_{3} ; \quad\left[e_{1}, e_{3}\right]=\left[e_{2}, e_{3}\right]=0
$$

The Lie algebra of Killing vector field of $\left(\mathbb{H}_{3}, g\right)$ is generated by the following killing vectors

$$
\begin{aligned}
& \mathbf{K}_{1}=\partial z, \quad \mathbf{K}_{2}=\partial y, \quad \mathbf{K}_{3}=\partial x-y \partial z \text { and } \\
& \mathbf{K}_{4}=\lambda^{2} y \partial x-x \partial y-\frac{1}{2}\left(\lambda^{2} y^{2}-x^{2}\right) \partial z
\end{aligned}
$$

and using the Eq. (7), we can rewrite the killing vectors in the base $\left(e_{i}\right)_{i=\overline{1,3}}$ as

$$
\begin{aligned}
& \mathbf{K}_{1}=e_{3}, \quad \mathbf{K}_{2}=e_{1}+x e_{3}, \quad \mathbf{K}_{3}=\frac{1}{\lambda} e_{2}-y e_{3} \text { and } \\
& \mathbf{K}_{4}=-x e_{1}+\lambda y e_{2}-\frac{1}{2}\left(\lambda^{2} y^{2}-3 x^{2}\right) e_{3} .
\end{aligned}
$$

for more detail see ([3]).

On the other hand, for the contact form

$$
\eta=x d y+d z=\omega^{3}
$$

and the $(1,1)$-tensor $\varphi$ given in base $\left(e_{i}\right)_{i=\overline{1,3}}$ by

$$
\varphi\left(e_{1}\right)=e_{2} ; \varphi\left(e_{2}\right)=-e_{1} \text { and } \varphi\left(e_{3}\right)=0
$$


we have

$$
\begin{aligned}
\eta\left(e_{3}\right) & =1 ; \varphi^{2}(X)=-X+\eta(X) e_{3} \text { and } \\
g(\varphi(X), \varphi(Y)) & =g(X, Y)+\eta(X) \eta(Y)
\end{aligned}
$$

for any $X, Y \in \chi\left(\mathbb{H}_{3}\right)$ and $\xi=e_{3}$, the Heisenberg group $\left(\mathbb{H}_{3}, \varphi, \xi, \eta, g\right)$ is an almost contact manifold. Moreover, we have

$$
d \eta(X, Y)=g(X, \varphi(Y))
$$

then $\left(\mathbb{H}_{3}, \varphi, \xi, \eta, g\right)$ is a contact manifold and the fundamental 2-form $d \eta$ is closed and hence it defines a magnetic field. For more detail see ([13], [14, [4], [16]).

\section{$4 \quad$ Killing magnetic curves in $\mathbb{H}_{3}$}

In this section, we have used two computer softwares (Wolfram Mathematica and Scientific Workplace) to solve some differential systems and for curve figures.

Let $\gamma(t)=(x(t), y(t), z(t)): I \subset \mathbb{R} \rightarrow\left(\mathbb{H}_{3}, g\right)$ be a regular curve. Its speed curve is

$$
\gamma^{\prime}(t)=\mathbf{t}=\left(x^{\prime}(t), y^{\prime}(t), z^{\prime}(t)\right)
$$

from the Eq. (7), the speed vector $\mathbf{t}$ is expressed in the base $\left(e_{i}\right)_{i=\overline{1,3}}$ as

$$
\mathbf{t}=y^{\prime} e_{1}+\frac{x^{\prime}}{\lambda} e_{2}+\left(z^{\prime}+x y^{\prime}\right) e_{3}
$$

Using the connection formulas given in the Eq. (8), the covariant derivative of the speed vector $\mathbf{t}$ is

$$
\nabla_{\mathbf{t}} \mathbf{t}=\left(y^{\prime \prime}+x^{\prime}\left(z^{\prime}+x y^{\prime}\right)\right) e_{1}+\left(\frac{x^{\prime \prime}}{\lambda}+\lambda y^{\prime}\left(z^{\prime}+x y^{\prime}\right)\right) e_{2}+\left(z^{\prime}+x y^{\prime}\right)^{\prime} e_{3}
$$

We know that the equation of geodesics is a particular case of the Lorentz equation given in Eq. 22 when the Lorentz force vanishes, the geodesics is a particular magnetic trajectories. A simple computation of the geodesic equation $\nabla_{\mathbf{t}} \mathbf{t}=0$, give a differential equations system

$$
S_{G}:\left\{\begin{array}{l}
y^{\prime \prime}+x^{\prime}\left(z^{\prime}+x y^{\prime}\right)=0 \\
x^{\prime \prime}+\lambda^{2} y^{\prime}\left(z^{\prime}+x y^{\prime}\right)=0 \\
\left(z^{\prime}+x y^{\prime}\right)^{\prime}=0
\end{array}\right.
$$

where its general solutions are explicit formulas of geodesic curves $\gamma_{G}$ in $\left(\mathbb{H}_{3}, g\right)$ given by the following proposition. 
Proposition 1 The parametric equations of the geodesic curves $\gamma_{G}$ in $\left(\mathbb{H}_{3}, g\right)$ is given by

$$
\begin{aligned}
& \text { i. } \gamma_{G}(t)=\left\{\begin{array}{l}
x(t)=c_{1} t+c_{2} \\
y(t)=c_{3} t+c_{4} \\
z(t)=\frac{c_{1} c_{3}}{2} t^{2}+c_{2} c_{3} t+c_{5}
\end{array}\right. \text { or } \\
& \text { ii. } \gamma_{G}(t)=\left\{\begin{array}{l}
x(t)=-\frac{c_{1}}{c} e^{c \lambda t}-\frac{c_{2}}{c} e^{-c \lambda t}+c_{3} \\
y(t)=\frac{c_{1}}{c \lambda} e^{c \lambda t}-\frac{c_{2}}{c \lambda} e^{-c \lambda t}+c_{4} \\
z(t)=\frac{2 c_{1} c_{2}+c^{2}}{c} t-\frac{c 3}{\lambda c}\left(c_{1} e^{c t \lambda}-c_{2} e^{-c t \lambda}\right)+\left(\frac{c_{1}^{2}-c_{2}^{2}}{2 c^{2} \lambda}\right) e^{-2 c \lambda t}
\end{array}\right.
\end{aligned}
$$

where $c_{\overline{1,4}}$ are a real constants.

\section{1 $\mathrm{K}_{1}$ - magnetic curves}

We consider $\mathbf{K}_{1}$-magnetic curves which correspond to the Killing vector field $\mathbf{K}_{1}=e_{3}=\partial z$.

Firstly, we have the product vector

$$
\mathbf{K}_{1} \times \mathbf{t}=-\frac{x^{\prime}}{\lambda} e_{1}+y^{\prime} e_{2}
$$

We can also obtain the Eq. (18) in the following way.

Because $\mathbf{K}_{1}=\xi$ given from the almost contact structure $\left(\mathbb{H}_{3}, \varphi, \xi, \eta, g\right)$, the magnetic fields $F_{\mathbf{K}_{1}}$ corresponding to $\mathbf{K}_{1}$ is exactly the magnetic fields $F$ associated to LORENTz force which presented by the skew-symmetric $(1,1)$-tensor $\varphi$ given by the Eq. 12.

Thus, we find the right-hand side of the relation given in Eq. 18.

$$
\begin{aligned}
\varphi(\mathbf{t}) & =\varphi\left(y^{\prime} e_{1}+\frac{x^{\prime}}{\lambda} e_{2}+\left(z^{\prime}+x y^{\prime}\right) e_{3}\right) \\
& =y^{\prime} e_{2}-\frac{x^{\prime}}{\lambda} e_{1}=\mathbf{K}_{1} \times \mathbf{t}
\end{aligned}
$$

Now, to find the explicit formulas of $\mathbf{K}_{1}$-magnetic curves, we must solve the differential equations system denoted by $\left(S_{1}\right)$, given from the Eqs. 16 . 18) and LORENTz equation

$$
\nabla_{\mathbf{t}} \mathbf{t}=\mathbf{K}_{1} \times \mathbf{t}
$$

The system $\left(S_{1}\right)$ is

$$
S_{1}:\left\{\begin{array}{l}
y^{\prime \prime}+x^{\prime}\left(\left(z^{\prime}+x y^{\prime}\right)+\frac{1}{\lambda}\right)=0 \\
x^{\prime \prime}+y^{\prime}\left(\lambda^{2}\left(z^{\prime}+x y^{\prime}\right)-\lambda\right)=0 \\
\left(z^{\prime}+x y^{\prime}\right)^{\prime}=0
\end{array}\right.
$$


After integrating the third equation $\left(S_{1_{3}}\right)$ and replaced them in the equations $\left(S_{1_{1,2}}\right)$, the system $(S)$ turns to

$$
S_{1}:\left\{\begin{array}{l}
y^{\prime \prime}+x^{\prime}\left(c+\frac{1}{\lambda}\right)=0 \\
x^{\prime \prime}+y^{\prime} \lambda(\lambda c-1)=0 \\
\left.z^{\prime}+x y^{\prime}=c \quad \text { constant }\right)
\end{array}\right.
$$

the general solution of the equations $\left(S_{1_{1,2}}\right)$ is

$$
\left\{\begin{array}{c}
x(t)=\frac{\lambda}{\lambda c+1}\left(c_{1} e^{\sqrt{\lambda^{2} c^{2}-1} t}+c_{2} e^{-\sqrt{\lambda^{2} c^{2}-1} t}\right)+c_{3} \\
y(t)=\frac{1}{\sqrt{\lambda^{2} c^{2}-1}}\left(c_{1} e^{\sqrt{\lambda^{2} c^{2}-1} t}-c_{2} e^{-\sqrt{\lambda^{2} c^{2}-1} t}\right)+c_{4}
\end{array}\right.
$$

where $c_{\overline{1,4}}$ are a real constants, $|\lambda c|>1$ (if $|\lambda c|<1$ the system don't admit a real solutions) and $\lambda c \neq \pm 1$ (we studied these cases $\lambda c= \pm 1$ later).

Substituting the Eq. 19$)$ in the equation $\left(S_{1_{3}}\right)$ and by an integration with respect to $t$, we have

$z(t)=\left(c+\frac{2 \lambda}{\lambda c+1} c_{1} c_{2}\right) t+\frac{1}{\sqrt{\lambda^{2} c^{2}-1}}\left(\begin{array}{c}c_{1} c_{3} e^{-t \sqrt{\lambda^{2} c^{2}-1}}-c_{2} c_{3} e^{t \sqrt{\lambda^{2} c^{2}-1}}+ \\ \frac{\lambda}{2(\lambda c+1)}\left(c_{1}^{2} e^{-2 t \sqrt{\lambda^{2} c^{2}-1}}-c_{2}^{2} e^{2 t \sqrt{\lambda^{2} c^{2}-1}}\right)\end{array}\right)+c_{5}$

where $c_{5}$ is real constant.

If $c=\frac{1}{\lambda}$, we rewrite the system $\left(S_{1}\right)$ as

$$
S_{1}:\left\{\begin{array}{l}
y^{\prime \prime}+x^{\prime} \frac{2}{\lambda}=0 \\
x^{\prime \prime}=0 \\
z^{\prime}+x y^{\prime}=\frac{1}{\lambda}
\end{array}\right.
$$

Its general solution is

$$
\left\{\begin{array}{l}
x(t)=c_{1} t+c_{2} \\
y(t)=\frac{-c_{1}}{\lambda} t^{2}+c_{3} t+c_{4} \\
z(t)=\frac{2 c_{1}^{2}}{3 \lambda} t^{3}+c_{1}\left(\frac{c_{2}}{\lambda}-\frac{c_{3}}{2}\right) t^{2}+\left(\frac{1}{\lambda}-c_{2} c_{3}\right) t+c_{5}
\end{array}\right.
$$

where $c_{\overline{1,5}}$ are a real constants.

If $c=\frac{-1}{\lambda}$, the system $\left(S_{1}\right)$ is

$$
S_{1}:\left\{\begin{array}{l}
y^{\prime \prime}=0 \\
x^{\prime \prime}-2 y^{\prime} \lambda=0 \\
z^{\prime}+x y^{\prime}=\frac{-1}{\lambda}
\end{array}\right.
$$


with a general solution

$$
\left\{\begin{array}{l}
x(t)=c_{1} \lambda t^{2}+c_{2} t+c_{3} \\
y(t)=c_{1} t+c_{4} \\
z(t)=\frac{c_{1} c_{2}}{2} t^{2}+\left(\frac{-1}{\lambda}+c_{3} c_{1}-\frac{c_{1}^{2}}{3} \lambda\right) t+c_{5}
\end{array}\right.
$$

Now, we can present the following theorem.

Theorem 2 The explicit formulae of all $\mathbf{K}_{1}$-magnetic curves in $\left(\mathbb{H}_{3}, g\right)$ are the space curves given by parametric equations:

1 .

$\gamma(t)=\left(\begin{array}{l}x(t)=c_{1} t+c_{2} \\ y(t)=\frac{-c_{1}}{\lambda} t^{2}+c_{3} t+c_{4} \\ z(t)=\frac{2 c_{1}^{2}}{3 \lambda} t^{3}+c_{1}\left(\frac{c_{2}}{\lambda}-\frac{c_{3}}{2}\right) t^{2}+\left(\frac{1}{\lambda}-c_{2} c_{3}\right) t+c_{5}\end{array}\right)$ or

$\gamma(t)=\left(\begin{array}{l}x(t)=c_{1} \lambda t^{2}+c_{2} t+c_{3} \\ y(t)=c_{1} t+c_{4} \\ z(t)=\frac{c_{1} c_{2}}{2} t^{2}+\left(\frac{-1}{\lambda}+c_{3} c_{1}-\frac{c_{1}^{2}}{3} \lambda\right) t+c_{5}\end{array}\right)$ or

3.

$\gamma(t)=\left(\begin{array}{l}x(t)=\frac{\lambda}{\lambda c+1}\left(c_{1} e^{\sqrt{\lambda^{2} c^{2}-1} t}+c_{2} e^{-\sqrt{\lambda^{2} c^{2}-1} t}\right)+c_{3} \\ y(t)=\frac{1}{\sqrt{\lambda^{2} c^{2}-1}}\left(c_{1} e^{\sqrt{\lambda^{2} c^{2}-1} t}-c_{2} e^{-\sqrt{\lambda^{2} c^{2}-1} t}\right)+c_{4} \\ z(t)=\left(c+\frac{2 \lambda c_{1} c_{2}}{\lambda c+1}\right) t+\frac{1}{\sqrt{\lambda^{2} c^{2}-1}}\left(\begin{array}{c}c_{3}\left(c_{1} e^{-t \sqrt{\lambda^{2} c^{2}-1}}-c_{2} e^{t \sqrt{\lambda^{2} c^{2}-1}}\right)+ \\ \frac{\lambda}{2(\lambda c+1)}\left(c_{1}^{2} e^{-2 t \sqrt{\lambda^{2} c^{2}-1}}-c_{2}^{2} e^{2 t \sqrt{\lambda^{2} c^{2}-1}}\right)\end{array}\right)+c_{5}\end{array}\right)$

where in (3), $|\lambda c|>1$ and $c, c_{\overline{1,5}}$ are a real constants.

Corollary 3 The curve presented in assertion (1) of the Theorem (2) for $c_{1}=0$ and $\lambda=\frac{1}{2 c_{2} c_{3}}$ is a geodesic $\mathbf{K}_{1}$-magnetic curve in $\left(\mathbb{H}_{3}, g\right)$.

Proof. It's a direct consequence from the geodesic curve formulas in Eq. 17) an the Theorem (2).

We present in $\left(\mathbb{R}_{3}, g_{\text {euc }}\right)$ some examples of $\mathbf{K}_{1}$-magnetic curve in $\left(\mathbb{H}_{3}, g\right)$ in following figures drawing in $\left(\mathbb{R}_{3}, g_{\text {euc }}\right)$.

For $c_{1,2.3}=1 ; \quad c_{4,5}=0$ and $\lambda=1$, the figure 1 at left side, present the $\mathbf{K}_{1}$-magnetic curve for the first assertion in $\left(\mathbb{H}_{3}, g\right)$.

For $c_{1,2}=\lambda=1$ and $c_{3,4,5}=0$, the figure 1 at right side, present the $\mathbf{K}_{1}$ magnetic curve for the assertion $(2)$ in $\left(\mathbb{H}_{3}, g\right)$.

For $c_{1,2,3}=1 ; \quad c_{4,5}=0$ and $\lambda=1 ; c=\sqrt{2}$ (i.e. $|\lambda c|>1$ ), the figure 2 present the $\mathbf{K}_{1}$-magnetic curve for the assertion (3) in $\left(\mathbb{H}_{3}, g\right)$. 


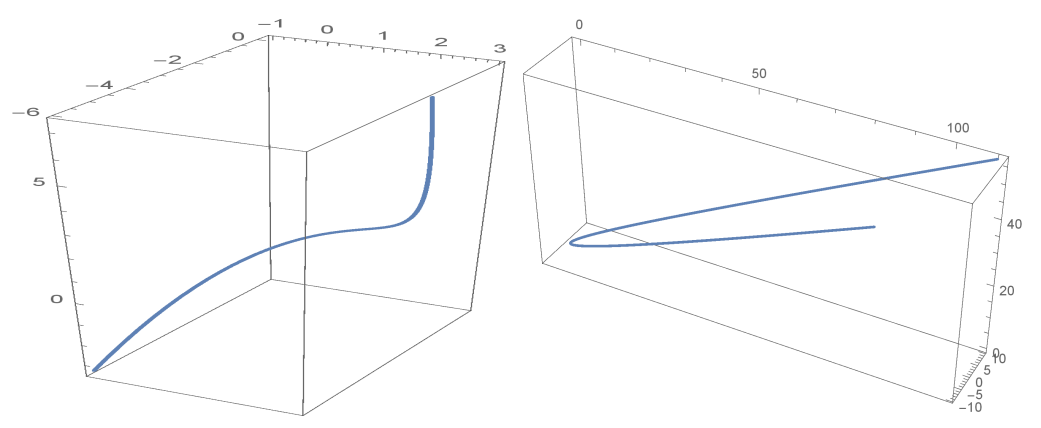

Figure 1: $\mathbf{K}_{1}$-magnetic curve

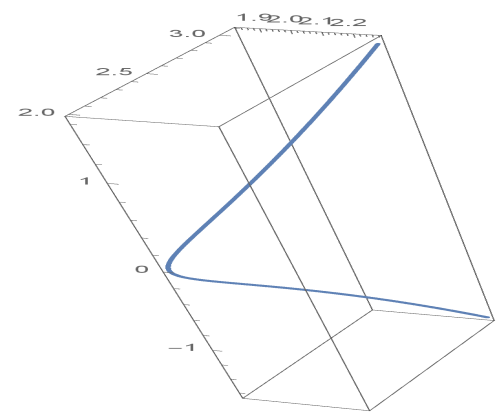

Figure 2: $\mathbf{K}_{1}$-magnetic curve

\section{$4.2 \quad \mathrm{~K}_{2}$-magnetic curves}

Similarly, as in subsection 4.1, we have

$$
\mathbf{K}_{2} \times \mathbf{t}=-\frac{x^{\prime} x}{\lambda} e_{1}+\left(x y^{\prime}-\left(z^{\prime}+x y^{\prime}\right)\right) e_{2}+\frac{x^{\prime}}{\lambda} e_{3}
$$

We can find a same formula as the Eq. (20) using the Eqs. (3, 4 and 11) to determine the magnetic fields $F_{\mathbf{K}_{2}}$ and associated skew-symmetric $(1,1)$ tensor $\varphi$.

The $\mathbf{K}_{2}$-magnetic curves are the solution of the differential equations system

$$
S_{2}:\left\{\begin{array}{l}
x^{\prime}\left(z^{\prime}+x y^{\prime}\right)=-y^{\prime \prime}-\frac{x x^{\prime}}{\lambda} \\
\left(\lambda y^{\prime}+1\right)\left(z^{\prime}+x y^{\prime}\right)=x y^{\prime}-\frac{x^{\prime \prime}}{\lambda} \\
\left(z^{\prime}+x y^{\prime}\right)^{\prime}=\frac{x^{\prime}}{\lambda}
\end{array}\right.
$$

given from the Eqs. 16, 20, and LORENTz equation

$$
\nabla_{\mathbf{t}} \mathbf{t}=\mathbf{K}_{2} \times \mathbf{t}
$$


after integrating the equation $\left(S_{2_{3}}\right)$, we have

$$
z^{\prime}+x y^{\prime}=\frac{x}{\lambda}+c
$$

here $c$ is a arbitrary real constant. Substituting the last equation in the equations $\left(S_{2_{2,3}}\right)$, we get the system

$$
\bar{S}_{2}:\left\{\begin{array}{l}
x^{\prime}\left(\frac{2 x}{\lambda}+c\right)=-y^{\prime \prime} \\
\left(\lambda y^{\prime}+1\right)\left(\frac{x}{\lambda}+c\right)=x y^{\prime}-\frac{x^{\prime \prime}}{\lambda}
\end{array}\right.
$$

and

$$
\bar{S}_{2}:\left\{\begin{array}{l}
y^{\prime}=-\frac{x^{2}}{\lambda}-x c \\
x^{\prime \prime}-c x^{2} \lambda+x\left(1-c^{2} \lambda^{2}\right)+c \lambda=0 .
\end{array}\right.
$$

it is very difficult to find exact solutions of the system $\left(\bar{S}_{2}\right)$ for every constant $c$. However, without loss of generality, we can assume that $c=0$, the solution can be given as

$$
\left\{\begin{array}{l}
x(t)=c_{1} \cos t-c_{2} \sin t \\
y(t)=\frac{1}{2 \lambda} c_{1} c_{2} \cos 2 t+\frac{1}{4 \lambda}\left(c_{1}^{2}-c_{2}^{2}\right) \sin 2 t+\frac{1}{2 \lambda}\left(c_{1}^{2}+c_{2}^{2}\right) t
\end{array}\right.
$$

Substituting the solutions $x(t)$ and $y(t)$ in Eq.21 and by an integration, we have the following solution

$$
z(t)=\frac{1}{\lambda}\left(\begin{array}{c}
\frac{1}{4} c_{2}\left(c_{1}^{2}-\frac{1}{3} c_{2}^{2}\right) \cos 3 t+\frac{1}{4} c_{1}\left(\frac{1}{3} c_{1}^{2}-c_{2}^{2}\right) \sin 3 t \\
+\left(1+\frac{3}{4}\left(c_{2}^{2}+c_{1}^{2}\right)\right)\left(c_{2} \cos t+c_{1} \sin t\right)
\end{array}\right)
$$

Then we have the following theorem:

Theorem 4 The space curves given by parametric equations

$$
\gamma(t)=\left\{\begin{array}{l}
x(t)=c_{1} \cos t-c_{2} \sin t \\
y(t)=\frac{1}{2 \lambda} c_{1} c_{2} \cos 2 t+\frac{1}{4 \lambda}\left(c_{1}^{2}-c_{2}^{2}\right) \sin 2 t+\frac{1}{2 \lambda}\left(c_{1}^{2}+c_{2}^{2}\right) t \\
z(t)=\frac{1}{\lambda}\left(\begin{array}{c}
\frac{1}{4} c_{2}\left(c_{1}^{2}-\frac{1}{3} c_{2}^{2}\right) \cos 3 t+\frac{1}{4} c_{1}\left(\frac{1}{3} c_{1}^{2}-c_{2}^{2}\right) \sin 3 t \\
+\left(1+\frac{3}{4}\left(c_{2}^{2}+c_{1}^{2}\right)\right)\left(c_{2} \cos t+c_{1} \sin t\right)
\end{array}\right)
\end{array}\right.
$$

are $\mathbf{K}_{2}$-Killing magnetic curves in $\left(\mathbb{H}_{3}, g\right)$, where $c_{1}, c_{2}$ are a real constants.

Corollary $\mathbf{5}$ There is no $\mathbf{K}_{2}$-magnetic curves od the space curves of the Theorem (4) which is a geodesic in $\left(\mathbb{H}_{3}, g\right)$.

In $\left(\mathbb{R}_{3}, g_{\text {euc }}\right)$, we present an example of $\mathbf{K}_{2}$-magnetic curve in $\left(\mathbb{H}_{3}, g\right)$ in figure 3 with $c_{1}=2 c_{2}=2$ and $\lambda=1$. 


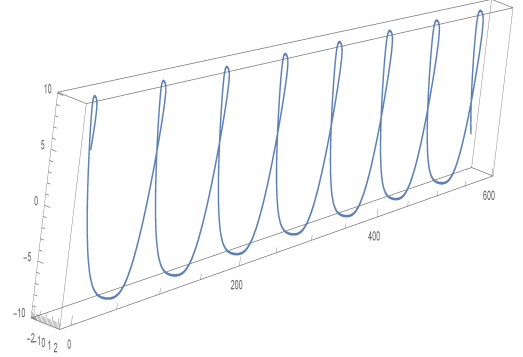

Figure 3: $\mathbf{K}_{2}$-magnetic curve in $\left(\mathbb{H}_{3}, g\right)$ presented in $\left(\mathbb{R}_{3}, g_{\text {euc }}\right)$

\section{$4.3 \quad K_{3}$-magnetic curves}

As above in subsections 4.1 and 4.2 , we have the product vector

$$
\mathbf{K}_{3} \times \mathbf{t}=\frac{1}{\lambda}\left(y x^{\prime}+z^{\prime}+x y^{\prime}\right) e_{1}-y y^{\prime} e_{2}-\frac{1}{\lambda} y^{\prime} e_{3}
$$

Also, we can find a same formula as the Eq. 20) using the Eqs.(3), 4 and 11) to determine the magnetic fields $F_{\mathbf{K}_{2}}$ and the associated skew-symmetric (1,1)-tensor $\varphi$.

Using the Eqs 16, 20 and

$$
\nabla_{\mathbf{t}} \mathbf{t}=\mathbf{K}_{3} \times \mathbf{t}
$$

we have the differential equations system $\left(S_{3}\right)$

$$
S_{3}:\left\{\begin{array}{c}
y^{\prime \prime}+x^{\prime}\left(z^{\prime}+x y^{\prime}\right)=\frac{1}{\lambda}\left(z^{\prime}+x y^{\prime}\right)+y \frac{x^{\prime}}{\lambda} \\
\frac{x^{\prime \prime}}{\lambda}+\lambda y^{\prime}\left(z^{\prime}+x y^{\prime}\right)=-y y^{\prime} \\
\left(z^{\prime}+x y^{\prime}\right)^{\prime}=-\frac{1}{\lambda} y^{\prime}
\end{array}\right.
$$

The integration of the third equation $\left(S_{3_{3}}\right)$, give

$$
z^{\prime}+x y^{\prime}=-\frac{1}{\lambda} y+c
$$

substituting the last equation in equations $\left(S_{3_{1,2}}\right)$, we have the differential equations system

$$
\bar{S}_{3}:\left\{\begin{array}{c}
y^{\prime \prime}+2 \lambda c y^{2}+y\left(\frac{1}{\lambda^{2}}-\lambda^{2} c^{2}\right)-\frac{c}{\lambda}=0 \\
x^{\prime}=-\lambda^{2} c y
\end{array}\right.
$$


Analogously as the subsection 4.2, it is a true challenge to find exact solutions for the system $\left(\bar{S}_{3}\right)$. Therefore, we try to solve'it in particular case $c=0$.

By integrating the system $\left(\bar{S}_{3}\right)$ in the case $c=0$, we find the general solution

$$
\left\{\begin{array}{l}
x(t)=c_{1} t+c_{2} \\
y(t)=c_{3} \exp \left(\frac{\sqrt{2 \lambda c_{1}-1}}{\lambda} t\right)+c_{4} \exp \left(\frac{-\sqrt{2 \lambda c_{1}-1}}{\lambda} t\right)
\end{array}\right.
$$

Substituting the solutions $x(t)$ and $y(t)$ in the Eq. 22, we get

$$
z(t)=\begin{gathered}
\left(\frac{-c_{3}}{\sqrt{2 \lambda c_{1}-1}}-c_{3}\left(c_{2}-\lambda c_{1} \sqrt{2 \lambda c_{1}-1}+c_{1} t\right)\right) \exp \left(\frac{\sqrt{2 \lambda c_{1}-1}}{\lambda} t\right) \\
+\left(\frac{c_{4}}{\sqrt{2 \lambda c_{1}-1}}-c_{4}\left(c_{2}+\lambda c_{1} \sqrt{2 \lambda c_{1}-1}+c_{1} t\right)\right) \exp \left(-\frac{\sqrt{2 \lambda c_{1}-1}}{\lambda} t\right)
\end{gathered}
$$

where $c_{\overline{1,4}}$ are an arbitrary real constants.

Finally, we can present the following theorem.

Theorem 6 The space curves given by parametric equations

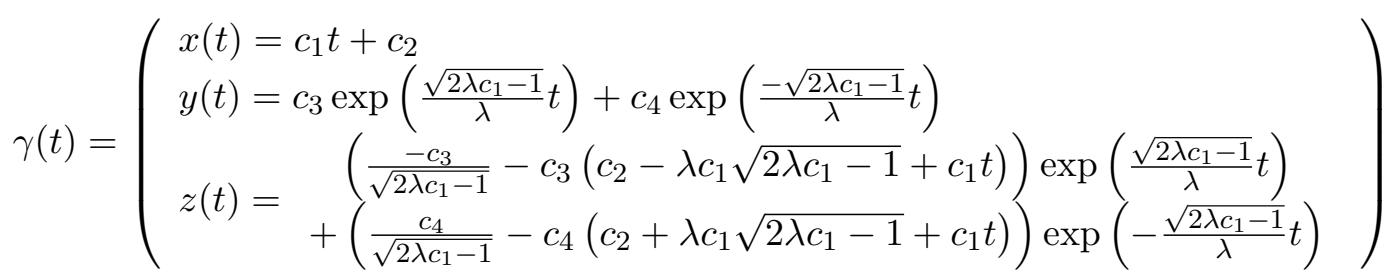

are $\mathbf{K}_{3}$-magnetic curves in $\left(\mathbb{H}_{3}, g\right)$, where $c_{\overline{1,4}}$ are a real constants.

Corollary $\mathbf{7}$ There is no $\mathbf{K}_{3}$-magnetic curves of the space curves of the Theorem (6) which is a geodesic in $\left(\mathbb{H}_{3}, g\right)$.

The following figure present in $\left(\mathbb{R}_{3}, g_{\text {euc }}\right)$ an example of $\mathbf{K}_{3}$-magnetic curve in $\left(\mathbb{H}_{3}, g\right)$ with $c_{\overline{1,4}}=1$ and $\lambda=1$. 


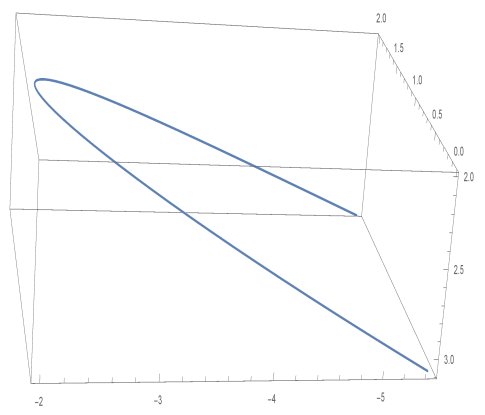

Figure 4: $\mathbf{K}_{3}$-magnetic curve in $\left(\mathbb{H}_{3}, g\right)$ presented in $\left(\mathbb{R}_{3}, g_{\text {euc }}\right)$

\section{$4.4 \quad K_{4}$-magnetic curves}

Finally, as subsections 4.1 , 4.2 and 4.3 , we consider $\mathbf{K}_{4}$-magnetic curves which correspond to the Killing vector field $\mathbf{K}_{4}$.

Firstly, we have the product vector

$$
\begin{aligned}
\mathbf{K}_{4} \times \mathbf{t}= & -\frac{1}{2 \lambda}\left(3 x^{2} x^{\prime}-y^{2} x^{\prime} \lambda^{2}-2 y \lambda^{2}\left(z^{\prime}+x y^{\prime}\right)\right) e_{1} \\
& -\frac{1}{2}\left(-3 x^{2} y^{\prime}+y^{2} y^{\prime} \lambda^{2}-2 x\left(z^{\prime}+x y^{\prime}\right)\right) e_{2}-\frac{1}{\lambda}\left(x x^{\prime}+y y^{\prime} \lambda^{2}\right) e_{3}
\end{aligned}
$$

The same formula can be found as the Eq. 23$)$, using the Eqs. 3 , 4 and 11) to determine the magnetic fields $F_{\mathbf{K}_{4}}$ and the associated skew-symmetric $(1,1)$-tensor $\varphi$.

Using the Eqs 16,23 and LoRENTz equation formula

$$
\nabla_{\mathbf{t}} \mathbf{t}=\mathbf{K}_{4} \times \mathbf{t}
$$

we have the differential equations system $\left(S_{4}\right)$

$$
S_{4}:\left\{\begin{array}{l}
y^{\prime \prime}+x^{\prime}\left(z^{\prime}+x y^{\prime}\right)=\frac{3}{2 \lambda} x^{2} x^{\prime}-\frac{\lambda}{2} y^{2} x^{\prime}-\lambda y\left(z^{\prime}+x y^{\prime}\right) \\
x^{\prime \prime}+\lambda^{2} y^{\prime}\left(z^{\prime}+x y^{\prime}\right)=\frac{3 \lambda}{2} x^{2} y^{\prime}-\frac{\lambda^{3}}{2} y^{2} y^{\prime}+\lambda x\left(z^{\prime}+x y^{\prime}\right) \\
\left(z^{\prime}+x y^{\prime}\right)^{\prime}=-\lambda y y^{\prime}-x \frac{x^{\prime}}{\lambda}
\end{array}\right.
$$

the integration of the third equation $\left(S_{4_{3}}\right)$, give

$$
z^{\prime}+x y^{\prime}=-\frac{x^{2}}{2 \lambda}-\frac{\lambda}{2} y^{2}+c
$$

Substituting the last equation in the equations $\left(S_{4_{1,2}}\right)$, we get

$$
\bar{S}_{4}:\left\{\begin{array}{l}
y^{\prime \prime}-\frac{2}{\lambda} x^{2} x^{\prime}=\frac{1}{2} y\left(y^{2} \lambda^{2}+x^{2}\right)-c\left(y \lambda+x^{\prime}\right) \\
x^{\prime \prime}-2 y^{\prime} x^{2} \lambda=-\frac{1}{2} x\left(y^{2} \lambda^{2}+x^{2}\right)+c\left(x \lambda-c y^{\prime} \lambda^{2}\right)
\end{array}\right.
$$


It is not essay to solve the last differential equations system $(\bar{S})$ or is not exactly solvable in general case, however we can solve'it in particular case when $c=0$ and let $x(t)=y(t)$ and taking account that $y^{2} \lambda^{2}+x^{2} \neq 0$, then $\left(\bar{S}_{4}\right)$ terns to

$$
y^{\prime \prime}-\frac{1+\lambda^{2}}{\lambda} y^{2} y^{\prime}=0
$$

by integrating the last equation with respect to $y$, we have

$$
y^{\prime}-\frac{1+\lambda^{2}}{3 \lambda} y^{3}=c_{1}
$$

where $c_{1}$ is a real constant. Without loss of generality, we can assume that $c_{1}=0$, then the non null solution is

$$
y(t)= \pm \frac{1}{\sqrt{2} \sqrt{c_{2}-\left(\frac{1+\lambda^{2}}{3 \lambda}\right) t}}
$$

where $c_{2}$ is a constant. From the Eq.(24), we have

$$
z(t)=\frac{3}{4} \ln \left|\left(\frac{1+\lambda^{2}}{3 \lambda}\right) t-c_{2}\right| \pm \frac{1}{4\left(c_{2}-\left(\frac{1+\lambda^{2}}{3 \lambda}\right) t\right)}+c_{3}
$$

where $c_{2}, c_{3}$ are a real constants. then we have the theorem.

Theorem 8 The space curves given by parametric equations

$$
\gamma(t)=\left(\begin{array}{rl}
x(t) & =\frac{1}{\sqrt{2} \sqrt{c_{1}-\left(\frac{1+\lambda^{2}}{3 \lambda}\right) t}} \\
y(t) & = \pm \frac{1}{\sqrt{2} \sqrt{c_{1}-\left(\frac{1+\lambda^{2}}{3 \lambda}\right) t}} \\
z(t) & =\frac{3}{4} \ln \left|\left(\frac{1+\lambda^{2}}{3 \lambda}\right) t-c_{1}\right| \pm \frac{1}{4\left(c_{1}-\left(\frac{1+\lambda^{2}}{3 \lambda}\right) t\right)}+c_{2}
\end{array}\right)
$$

are $\mathbf{K}_{4}$-magnetic curves in $\left(\mathbb{H}_{3}, g\right)$, where $c_{1}, c_{2}$ are an arbitrary real constants.

Corollary $\mathbf{9}$ There is no $\mathbf{K}_{4}$-magnetic curves of the space curves of the Theorem (8) which is a geodesic in $\left(\mathbb{H}_{3}, g\right)$.

Finally, we present in the following figure in $\left(\mathbb{R}_{3}, g_{\text {euc }}\right)$, an example of $\mathbf{K}_{4}$-magnetic curve in $\left(\mathbb{H}_{3}, g\right)$ where $c_{1}=c_{2}=0$ and $\lambda=1$. 


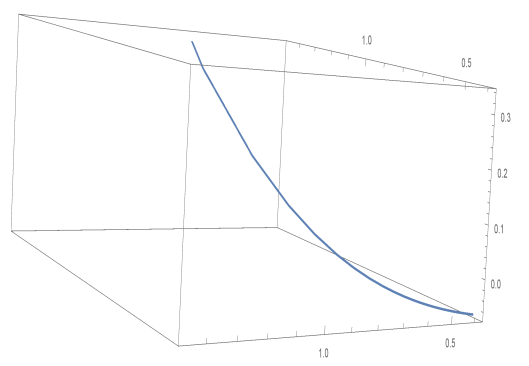

Figure 5: $\mathbf{K}_{4}$-magnetic curve in $\left(\mathbb{H}_{3}, g\right)$ presented in $\left(\mathbb{R}_{3}, g_{\text {euc }}\right)$

\section{References}

[1] M. Barros, A. Romero: Magnetic vortices, EPL 77 (2007), 34002.

[2] C. Bejan, S. L. Drută-Romaniuc. Walker manifolds and Killing magnetic curves, Diff. Geo. and its App. , 35 (2014), 106-116. doi.org/10.1016/j.difgeo.2014.03.001.

[3] W. Batat, A. Zaeim. On symmetries of the Heisenberg group, arXiv:1710.04539v1.

[4] L. Capogna, D. Danielli, S.D. Pauls, J.T. Tyson. An introduction to the Heisenberg group and the sub-Riemannian isoperimetric problem. (Progress in Mathematics). Birkhäuser Verlag, Basel (2007).

[5] G. Calvaruso, M. I. Munteanu, A. Perrone. Killing magnetic curves in three-dimensional almost paracontact manifolds, J. of Math. Ana. and App., 426(1), (2015), 423-439. doi.org/10.1016/j.jmaa.2015.01.057

[6] S. L. Drută-Romaniuc, J. Inoguchi, M. I. Munteanu and A. I. Nistor: Magnetic curves in cosymplectic manifolds, Rep. Math. Phys. 78 (2016), 33 .

[7] S. L. Drută-Romaniuc, J. Inoguchi, M. I. Munteanu, A. I. Nistor: Magnetic curves in Sasakian manifolds, J. Nonlinear Math. Phys. 22 (2015), 428 .

[8] S. L. Drută-Romaniuc, M. I. Munteanu. Killing magnetic curves in a Minkowski 3-space, Nonlinear Analysis: Real World Appl. 14 (2013), 383. 
[9] S. L. Drută-Romaniuc and M. I. Munteanu, Magnetic curves corresponding to Killing magnetic fields in $\mathbb{E}^{3}$, J. Math. Phys. 52 (2011), 113506.

[10] Z. Erjavec. On Killing magnetic curves in $S L(2, \mathbb{R})$ geometry, Reports on mathematical physics, 84(3), (2019), 333-350.

[11] Z. Erjavec, Ji. Inoguchi. On Magnetic Curves in Almost Cosymplectic Sol Space. Results Math 75, 113 (2020). doi.org/10.1007/s00025-02001235-y

[12] Z. Erjavec, Ji. Inoguchi. Killing Magnetic Curves in Sol Space. Math. Phys. Anal. Geom. 21, 15 (2018). doi.org/10.1007/s11040-018-9272-6

[13] C. Figueroa. The Gauss map of minimal graphs in the Hzisenberg group, J. of Geo. and Sym. in Phys. 25, (2012), 1-21.

[14] T. Hangan. Au sujet des flots riemanniens sur le groupe nilpotent de Heisenberg. Rend. Circ. Mat. Palermo 35, 291-305 (1986). doi.org/10.1007/BF02844738.

[15] Z. Iqbal, J. Sengupta, S. Chakraborty. Magnetic trajectories corresponding to Killing magnetic fields in a three-dimensional warped product 2020, Inter. J. of Geo. Meth. in Mod. Phys., 17(14), 2050212 (2020). doi.org/10.1142/S0219887820502126

[16] J. Milnor, Curvature of left invariant metrics on Lie groups, Adv. Math. 21 (1976), 293-329.

[17] M. I. Munteanua, A. Nistor. The classification of Killing magnetic curves in $\mathbb{S}^{2} \times \mathbb{R}$, J. of Geo. and Phys., 62 (2012) 170-182. doi:10.1016/j.geomphys.2011.10.002

[18] C. Özgür. On magnetic curves in 3-dimensional Heisenberg group, Pro. of the Ins. of Math. and Mech., National Academy of Sciences of Azerbaijan, 43(2), (2017), 278-286. 